\section{FEED BODY AND BRAIN}

Oral-B's popular 'lunch and learn' sessions are CPD accredited so all team members can earn one hour of verifiable CPD in the comfort of their own practice. The meetings are ideal for those who want to learn more about the latest developments in power brushing and toothpaste including clinical support and marketing opportunities. Samples for patient and personal use will also be made available.

Oral-B will endeavour to visit on the most convenient day for the practice and will provide a one hour presentation focusing on clinical data behind their products. As the presentation takes place at lunchtime, Oral-B will provide lunch for all team members present.

Access to the Internet has led to an increased interest and awareness amongst consumers regarding matters of oral hygiene, and it is hoped that these informal sessions will allow staff members to learn about Oral-B's products and ask any questions they might have in a relaxed environment with as little disruption as possible to their normal working day.

These events are popular and get booked up early. To request a 'lunch and learn' demonstration you will need to contact your local representative. If you do not have their details please call 08702421850 and a member of the customer service team will put you in contact. As demand is high, reps will allocate appointments on a 'first come, first served' basis.

\title{
EXTREMELY RESISTANT TO CAMERA SHAKE
}

CEREC Omnicam generates full colour scans of intraoral surfaces without the need for a powder coating.

Three features of the CEREC Omnicam stand out in particular: it supports video streaming; it digitises the structures of the jaw in their natural colour; and it does not require a powder coating of the tooth surfaces.

The CEREC Omnicam is extremely resistant to camera shake and it's not necessary to apply a powder coating to the teeth. Instead the user moves the camera head over the intraoral surfaces in a smooth, flowing movement. A virtual
3D model is displayed in natural colour on the monitor of the CEREC AC. Thanks to this lifelike visualisation, it is easy to distinguish between different materials and identify the palatinal and gingival contours. As a result it is easy to navigate around the oral cavity and determine the preparation margin.

The extensive depth of field of CEREC Omnicam delivers high-precision images, even in cases where the camera has been placed directly on the tooth. The ergonomic, lightweight camera feels comfortable in the hand. Thanks to the slimline design and the compact camera head, it is easy to scan inaccessible areas such as the distal surfaces of the posterior molars.

In addition, the CEREC Omnicam boasts a patient counselling mode. The dentist can record short video clips and present these to the patient via the CEREC AC monitor.

Call Sirona Dental Systems on 08450715040.

\section{SIMPLY FILL THE BOTTLE AND GO}

Compact yet powerful, the standalone Mectron Prophy Unit the Mectron Piezo Smart incorporates the latest technology and ergonomic design, enabling operators to concentrate solely on delivering optimised treatment, whether dealing with supra- or sub-gingival calculus, orthograde or retrograde endodontics or simple restorative treatment.

With its simple, safe and easy-to-use bottle system, the Mectron Piezo Smart enables operators to use various flushing solutions for optimised therapy without any risk of dripping or leaking. No need to plumb it in; simply fill the bottle, put it in place and off you go! Its easyto-use touch-screen controls enable operators to select the appropriate function (endo, perio or scaler) and irrigation flow rate.

The Mectron Piezo Smart features the latest ultrasound technology which recognises external influences and offsets them automatically to deliver constant optimum performance for every application, while its slimline handpiece is compatible with over 40 Mectron Inserts and fits comfortably in the hand.

Extremely competitively priced, the Mectron Piezo Smart is available for just $£ 1,451.74$ plus VAT.

For further information call 01380734990 or visit www. generalmedical.co.uk.
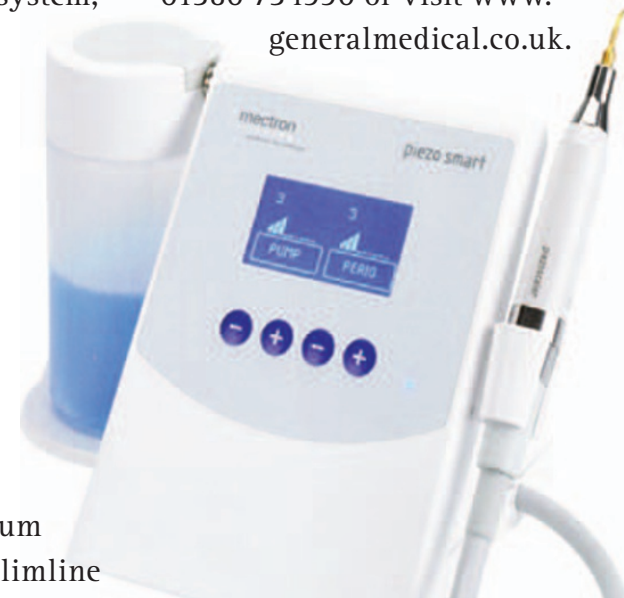

\section{INCREASE YOUR IMPLANT REFERRAL BASE}

United Smile Centres are looking to partner with a select number of implant practices throughout the UK, offering the United Smile Centres expertise, quality and patient care on a national scale.

Benefits include the potential to increase annual turnover by $20-40 \%$; extensive monthly consumer marketing; all patient enquiries will be pre-screened before being passed on to you; the support of an exceptional call centre team; the implant buying power of a large corporate; highest quality lab work at a fixed cost; and all the benefits of being part of an established effective group, but retaining control.

Call 08008494959 or visit www.unitedsmilecentres.co.uk. 